\title{
Multidetector computed tomography to detect reversible subclinical aortic bioprosthetic valve thrombosis with high systolic gradients
}

\author{
Rafał Gałąska ${ }^{1}$, Dorota Kulawiak-Gałąska ${ }^{2}$, Marcin Fijałkowski ${ }^{1}$, \\ Edyta Szurowska ${ }^{3}$, Marcin Gruchała ${ }^{1}$ \\ ${ }^{1} 1^{\text {st }}$ Department of Cardiology, Medical University of Gdansk, Poland \\ ${ }^{2}$ Department of Radiology, Medical University of Gdansk, Poland \\ ${ }^{3} 2^{\text {nd }}$ Department of Radiology, Medical University of Gdansk, Poland
}

A 64-year-old asymptomatic patient was admitted to the hospital due to high gradients of the biological aortic valve, which was diagnosed during a control transthoracic echocardiographic examination. The patient suffered from aortic valve regurgitation as a complication of infective endocarditis and he underwent aortic valve replacement with bioprosthesis 15 months before admission. Warfarin was started after cardiac surgery and was continued for 3 months. The therapy was then withdrawn and switched to acetylsalicylic acid (ASA). The highest systolic gradients of aortic bioprosthesis from echocardiographic reports during the first 6 months after surgery were $35 / 22 \mathrm{~mm} \mathrm{Hg}$ (maximum/mean systolic gradient). During hospitalization a transthoracic and transesophageal examinations were performed, which confirmed high systolic gradients (68.3/ /46.5 mm Hg; Fig. 1). The calculated effective valve area was $1.0 \mathrm{~cm}^{2}$. Due to dissatisfactory visualization of bioprosthesis leaflets on the echocardiography, the patient was referred to have cardiac multidetector computed tomography (MDCT). The MDCT showed that the basal part of the aortic bioprosthesis leaflets was thickened with limited mobility. Antithrombotic treatment with low-molecular-weight heparin was commenced, and after 10 days a slight decrease of gradient was observed (48.9/30.3 mm Hg). The patient was then switched to warfarin and discharged from the hospital. The aortic gradient decreased to $23.6 / 13.9 \mathrm{~mm} \mathrm{Hg}$ after 3 months of treatment. The calculated affective valve area after treatment was $1.4 \mathrm{~cm}^{2}$. During a control cardiac MDCT we observed a resolution of thrombotic changes with normal leaflet mobility and normal leaflet thickness.

Recently, Makkar et al. [1] reported an increased prevalence of bioprosthetic aortic valves thrombosis on MDCT examination. According to the authors, multiple bioprosthesis types were affected including the ones with transcatheter (TAVR) and surgically (AVR) implanted bioprostheses. The authors' preliminary report included patients from ongoing RESOLVE and SAVORY registries, as well as a PORTICO IDE randomized trial, which were started to establish the effect of this finding on clinical outcomes [2-4]. Affected patients appeared to be hemodynamically subclinical at the time of detection, with normal aortic valve gradients on their echocardiography. The median time to MDCT examination was significantly shorter after the AVR/TAVR procedure in comparison to our patient, who was asymptomatic but he had significantly increased aortic bioprosthesis gradients. It is possible that the valve thrombosis that we observed was more severe due to the longer period of time after valve replacement. According to the European Society of Cardiology guidelines anticoagulant therapy has been challenged in patients with aortic bioprosthesis, with the use of low-dose ASA now favoured as an alternative. Further randomized trials are needed to establish the clinical significance of the finding of Makkar et al. [1], but possibly biological valve thrombosis and its consequences due to insufficient antithrombotic treatment occur more frequently than had been previously assumed.

Address for correspondence: Rafał Gałąska, MD, PhD, $1^{\text {st }}$ Department of Cardiology, Medical University of Gdansk, ul. Dębinki 7, 80-952 Gdańsk, Poland, tel: +48 5834925 00; 34925 04, fax: +48 5834612 01, e-mail: rgal@gumed.edu.pl 


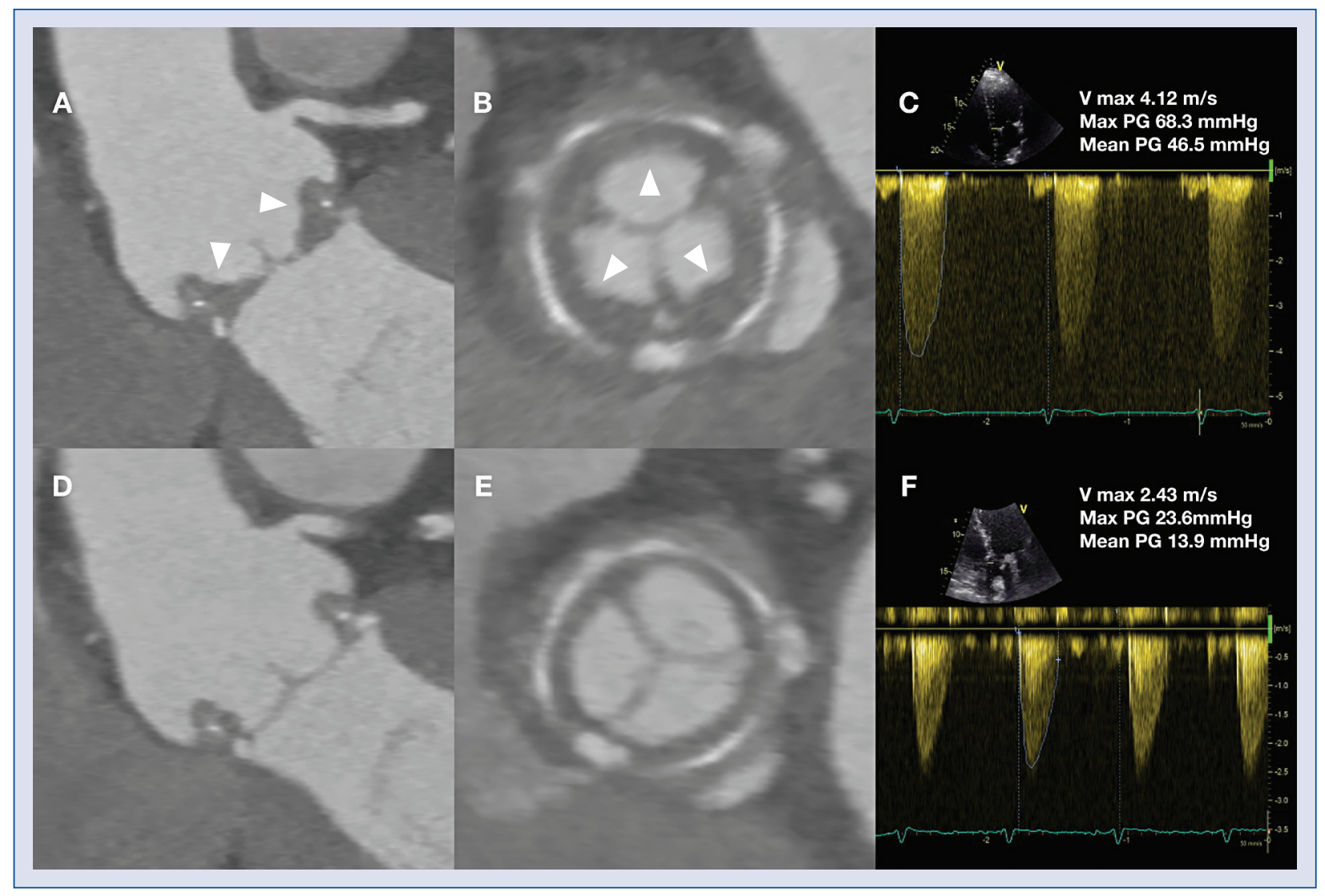

Figure 1. A, B. Multidetector computed tomography (MDCT) multiplanar reconstruction demonstrating thrombosed aortic bioprosthesis before antithrombotic treatment (arrowheads); C. Transthoracic echocardiography, continuous spectral Doppler imaging, and high aortic bioprosthesis systolic gradient before antithrombotic treatment; D, E. MDCT after treatment; F. Transthoracic echocardiography, decreased gradient after treatment.

\section{References}

1. Makkar RR, Fontana G, Jilaihawi $H$ et al. Possible subclinical leaflet thrombosis in bioprosthetic aortic valves. N Engl J Med, 2015; 373: 2015-2024.

2. The Assessment of Transcatheter and Surgical Aortic Bioprosthetic Valve Thrombosis and its Treatment with Anticoagulation (RESOLVE) registry (ongoing).
3. Subclinical Aortic Valve Bioprosthesis Thrombosis Assessed with Four-Dimensional Computed Tomography (SAVORY) registry (ongoing).

4. The Portico Resheathable Transcatheter Aortic Valve System U.S. Investigational Device Exemption (PORTICO IDE) study (ongoing).

Conflict of interest: None declared 